\title{
Xylitol Separation from a Polyol Mixture Using Lanthanide Ion-loaded Resins
}

\author{
Yusuke Kitamura, ${ }^{\dagger}$ Rika Shobu, Hirotaka Matsuura, Akinori Jyo, and Toshihiro IhaRa ${ }^{\dagger}$ \\ Division of Materials Science and Chemistry, Faculty of Advanced Science and Technology, Kumamoto University, \\ 2-39-1 Kurokami, Chuo, Kumamoto 860-8555, Japan
}

Xylitol separation from a polyol mixture of the byproducts from bioethanol production processes was performed by liquid chromatography using short columns packed with lanthanide ion-loaded ion-exchange resins. Xylitol was successfully separated with sufficiently high resolution using adsorbents with medium rare-earth metal ions, such as $\mathrm{Nd}^{3+}$ and $\mathrm{Sm}^{3+}$. The adsorbents' specific nature is explained by the so-called "gadolinium break," which is known as a discontinuous behavior of thermodynamic parameters in complexation of the lanthanide series. From the observed behavior, the optimum lanthanide ions could be chosen to prepare appropriate adsorbents for ligand-exchange chromatography of given polyol mixtures.

Keywords Monosaccharide, polyol, lanthanide, rare-earth metal, ligand exchange, bioethanol, gadolinium break, liquid chromatography, ion-exchange resin, AG 50W-X4

(Received November 28, 2019; Accepted December 26, 2019; Advance Publication Released Online by J-STAGE January 10, 2020)

\section{Introduction}

Bioethanol fuel is one of the petrol substitutes produced from agricultural crops by the sugar fermentation process. ${ }^{1}$ This fuel would be one of the solutions for the greenhouse effect and the depletion of fossil fuels; however, the large-scale production of bioethanol from cereal crops, such as corn and wheat, could cause water shortages as well as adverse effects for the food supply. The use of nonfood plants, waste materials, such as used straw and willow, sawdust, and even municipal solid wastes are therefore ongoing research targets as a hopeful feedstock for bioethanol. ${ }^{2-4}$

Bamboo invasion is a serious environmental issue in many Japanese cities. The disordered proliferation of moso bamboo (Phyllostachys edulis, a non-native species) due to a lack of control rapidly converts the deciduous and broadleaf forests into bamboo forests..$^{5-7}$ Prompt action against bamboo invasion is also necessary in Kumamoto, where an effective use of fallen bamboo trees has been explored. We have taken part in a local project of bioethanol production from bamboo. Ethanol can be produced from bamboo through hydrolysis and sugar fermentation processes. ${ }^{8,9}$ From our optimized processes using Candida tropicalis, three polyols, xylose, xylitol, and glycerol, are obtained as byproducts. Among them, xylitol is a highvalue chemical due to its use as a sweetener. ${ }^{10-14}$ However, a small amount of glycerol (b.p., $290^{\circ} \mathrm{C}$ ) interferes with the crystallization of xylitol because of its highly hygroscopic nature.

In the present study, we tried to separate the polyols by liquid chromatography. As the separation mode, here we chose ligand exchange on trivalent lanthanide ions $\left(\mathrm{Ln}^{3+}\right)$ loaded on cationexchange resins. A strong-acid cation exchanger quantitatively

† To whom correspondence should be addressed.

E-mail: toshi@chem.kumamoto-u.ac.jp captures $\mathrm{Ln}^{3+}$ and, in chromatography, the residual coordination room on the $\mathrm{Ln}^{3+}$ sphere on the other side of the resin could be used to retain the molecules with Lewis base atoms (Fig. 1). There are some merits of the ligand-exchange mode for the separation of biomolecules. ${ }^{15-21}$ Aqueous solutions (no organic solvent) of neutral $\mathrm{pH}$ are used as an eluent, which is ecofriendly. Appropriate metal ions could be chosen for each of the given mixtures of analytes on demand. Especially for $\mathrm{Ln}^{3+}$, while they show similarity in various chemical properties, the size of the

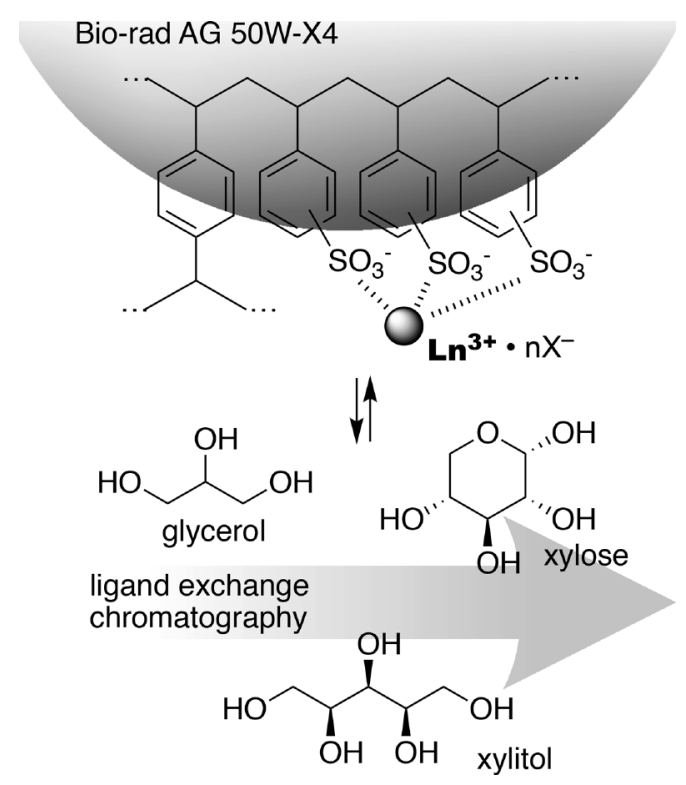

Fig. 1 Chemical structures of ion-exchange resin and analytes used for the study of polyol separation by ligand-exchange mode using $\mathrm{Ln}^{3+}$-loaded resin. 
Table 1 Ion-exchange parameters of $\mathrm{Ln}^{3+}$-type resin (AG 50W-X4; Bio-Rad)

\begin{tabular}{|c|c|c|c|c|c|c|c|c|}
\hline $\mathrm{Ln}^{3+}$ & & $\mathrm{La}$ & $\mathrm{Ce}$ & $\mathrm{Nd}$ & $\mathrm{Sm}$ & $\mathrm{Gd}$ & Ho & $\mathrm{Yb}$ \\
\hline Shrinkage $(S F), \%$ & & 30.1 & 31.2 & 29.9 & 32.1 & 30.6 & 31.1 & 26.7 \\
\hline $\begin{array}{l}\text { Ion-exchange capacity, } \\
\mathrm{meq} / \mathrm{mL}\end{array}$ & $\begin{array}{l}I X_{\max } \\
I X_{\mathrm{obs}}\end{array}$ & $\begin{array}{l}0.53 \\
0.535\end{array}$ & $\begin{array}{l}0.53 \\
0.520\end{array}$ & $\begin{array}{l}0.52 \\
0.494\end{array}$ & $\begin{array}{l}0.54 \\
0.513\end{array}$ & $\begin{array}{l}0.53 \\
0.471\end{array}$ & $\begin{array}{l}0.53 \\
0.473\end{array}$ & $\begin{array}{l}0.50 \\
0.411\end{array}$ \\
\hline Ion-exchange rate $(R I X), \%$ & & $\sim 100$ & 98 & 95 & 95 & 91 & 89 & 84 \\
\hline
\end{tabular}

ions decreases from $\mathrm{La}^{3+}$ to $\mathrm{Lu}^{3+}$, which is known as lanthanide contraction. We can prepare a series of $\mathrm{Ln}^{3+}$-loaded resins that could be subjected to a systematic study for the separation of analyte mixtures. Hydroxyl groups in polyols are the typical hard Lewis base. Therefore, the nature of $\mathrm{Ln}^{3+}$ as a hard Lewis acid should make the $\mathrm{Ln}^{3+}$-loaded resins excellent flexible adsorbents for polyols. ${ }^{22-24}$ In ligand-exchange chromatography, the $\mathrm{Ln}^{3+}$-loaded adsorbents are expected to retain the polyols depending on their structural parameters, such as the number of hydroxyl groups, stereochemistry, and flexibility of molecular backbones (Fig. 1).

\section{Experimental}

\section{Reagents and chemicals}

All materials and chemicals were of analytical grade and used without further purification. A strong-acid cation-exchange resin, AG 50W-X4 (hydrogen ion form; crosslinkage, 4\%; particle size, $0.075-0.150 \mathrm{~mm}$; total cation exchange capacity, $1.1 \mathrm{meq} / \mathrm{mL}$ ) was purchased from Bio-Rad Laboratories (Hercules, CA, USA). $\mathrm{LaCl}_{3} \cdot 6 \mathrm{H}_{2} \mathrm{O}, \mathrm{CeCl}_{3} \cdot 6 \mathrm{H}_{2} \mathrm{O}, \mathrm{SmCl}_{3} \cdot 6 \mathrm{H}_{2} \mathrm{O}$, $\mathrm{Gd}\left(\mathrm{NO}_{3}\right)_{3} \cdot 6 \mathrm{H}_{2} \mathrm{O}, \mathrm{HoCl}_{3} \cdot 6 \mathrm{H}_{2} \mathrm{O}$, and $\mathrm{YbCl}_{3} \cdot 6 \mathrm{H}_{2} \mathrm{O}$ were purchased from Wako Pure Chemical Industries (Osaka, Japan). $\mathrm{Nd}\left(\mathrm{NO}_{3}\right)_{3} \cdot 6 \mathrm{H}_{2} \mathrm{O}$ was purchased from Junsei Chemical (Tokyo, Japan). D-(+)-Xylose, xylitol, glycerol and all other chemicals used in this study were purchased from Wako Pure Chemical Industries. Ultra-pure water (Milli-Q, Integral MT5L; Nippon Millipore, Tokyo, Japan) was used for all experimental work.

\section{Preparation of $\mathrm{Ln}^{3+}-$ loaded resin}

Twenty milliliters of swollen AG 50W-X4 was washed by decantation with water several times, packed into a glass column (inner diameter, $12 \mathrm{~mm}$; length, $20 \mathrm{~cm}$ ), and then the column was subjected to Milli-Q water conditioning at a flow rate of $20 \mathrm{~mL} / \mathrm{h}$ for $3 \mathrm{~h}$. Then, $100 \mathrm{~mL}$ (5.0 BV [Bed volumes]) of an aqueous $\mathrm{LaCl}_{3}$ solution $(0.36 \mathrm{~mol} / \mathrm{L})$ was fed to the column at a flow rate of $20 \mathrm{~mL} / \mathrm{h}$. After washing the column with $100 \mathrm{~mL}$ of Milli-Q water, $\mathrm{La}^{3+}$-loaded adsorbent was subjected to the experiments for an measurement concerning the ion-exchange rate and polyol separation. Other adsorbents loaded with $\mathrm{Ce}^{3+}$, $\mathrm{Nd}^{3+}, \mathrm{Sm}^{3+}, \mathrm{Gd}^{3+}, \mathrm{Ho}^{3+}$, and $\mathrm{Yb}^{3+}$ were prepared similarly.

\section{Measurement of ion exchange rate}

The swollen volume of $\mathrm{Ln}^{3+}$-loaded resins $(c a .1 .0 \mathrm{~mL})$ was measured with a measuring cylinder after overnight settling. The resins were completely dried in a vacuum desiccator and their dry weights were measured. Certain amounts of the dried resins were completely liquefied with $4 \mathrm{~mL}$ of conc. $\mathrm{HNO}_{3}$ using a microwave digestion oven (Speedwave 4; Berghof Products Instruments, Berghof, Germany). Three samples were prepared for each of the adsorbents for duplicate measurements.

The digested solutions of each of the adsorbents were appropriately diluted to be around $8 \mathrm{ppm}$ for the concentration of $\mathrm{Ln}^{3+}$ and subjected to concentration measurements by ICPAES (inductively coupled plasma-atomic emission spectroscopy, Nippon Jarrell Ash, Kyoto, Japan). The ion-exchange capacities $\left(I X_{\text {obs }}[\mathrm{meq} / \mathrm{mL}]\right)$ of the resins for each of $\mathrm{Ln}^{3+}$ were estimated using respective standard curves. The ion-exchange rates (RIX [\%]) for each of $\mathrm{Ln}^{3+}$ were calculated as a relative value with the theoretical ion-exchange capacities $\left(I X_{\max }[\mathrm{meq} / \mathrm{mL}]\right.$, the maximum $\mathrm{Ln}^{3+}$ capacity loaded on the ion-exchange resin with $1.1 \mathrm{meq} / \mathrm{mL}$ acid capacity) estimated by following equation:

$$
\begin{aligned}
& I X_{\max }=\frac{1.1}{3} /\left(1-\frac{S F}{100}\right)[\mathrm{meq} / \mathrm{mL}], \\
& R I X=\left(\frac{I X_{\mathrm{obs}}}{I X_{\max }}\right) \times 100[\%],
\end{aligned}
$$

where the $S F[\%]$ is the shrinkage factor of the volume of resin when it fully loads $\mathrm{Ln}^{3+}$.

\section{Polyols separation}

Ten milliliters of each of the $\mathrm{Ln}^{3+}$-loaded resins were packed into a glass column (inner diameter, $12 \mathrm{~mm}$; length, $20 \mathrm{~cm}$ ), and the columns were subjected to Milli-Q water conditioning at a flow rate of $20 \mathrm{~mL} / \mathrm{h}$ for $3 \mathrm{~h}$. Then, $3 \mathrm{~mL}(0.3 \mathrm{BV})$ of a mixture of xylose, xylitol, and glycerol (20 g/L for each) was loaded to the columns at a flow rate of $20 \mathrm{~mL} / \mathrm{h}$. Milli-Q water was fed to the columns as an eluent at a flow rate of $20 \mathrm{~mL} / \mathrm{h}$. All effluents were collected on a fraction collector by $1.5 \mathrm{~mL}$ $(0.15 \mathrm{BV})$ for each fraction, which was analyzed with HPLC (Sugar Analyzer SU-300; DKK-TOA, Tokyo, Japan) after suitable dilution. The resolution $(R)$ of the two components was calculated from a chromatogram. The $R$ value was defined as $2\left(V_{\mathrm{R} 2}-V_{\mathrm{R} 1}\right) /\left(w_{\mathrm{b} 1}+w_{\mathrm{b} 2}\right)$, where $V_{\mathrm{R} 1}$ and $V_{\mathrm{R} 2}$ are the retention volumes of polyols 1 and 2 , and $w_{\mathrm{b} 1}$ and $w_{\mathrm{b} 2}$ are the peak widths (baseline) of polyols 1 and 2, respectively.

\section{Results and Discussion}

\section{Ion-exchange rate for the series of lanthanide ions}

Enormous knowledge concerning cation binding on various solid supports have been accumulated during a long history of studies on ion-exchange resins to date. We can now choose the suitable resin appropriate for our research purpose while considering several requirements regarding, for example, the types of cations (hard or soft) and how to use the resin (reversible or irreversible binding). ${ }^{25-28}$ Here, we chose AG 50W-X4, which is a strong-acid cation-exchange resin with sulfonic acid groups. Seven $\mathrm{Ln}^{3+}$ from the lanthanide series $\left(\mathrm{La}^{3+}, \mathrm{Ce}^{3+}, \mathrm{Nd}^{3+}, \mathrm{Sm}^{3+}\right.$, $\mathrm{Gd}^{3+}, \mathrm{Ho}^{3+}$, and $\mathrm{Yb}^{3+}$ ) were loaded on the resin using the column method. Table 1 gives some properties of the $\mathrm{Ln}^{3+}$-loaded resin regarding its ion-exchange capacities. The AG 50W-X4 resin significantly shrank after loading of $\mathrm{Ln}^{3+}$. We observed ca. 30\% $S F$ throughout a series of $\mathrm{Ln}^{3+}$. Trivalent cations would reduce 
the electrostatic repulsion between the sulfonate groups or, even more directly, convergently draw the distal two or three points on the polymer up to themselves by bridging the sulfonate groups. $^{29}$ Apparent particular trends, such as the lanthanide contraction in the $S F$, were not observed. RIX was calculated

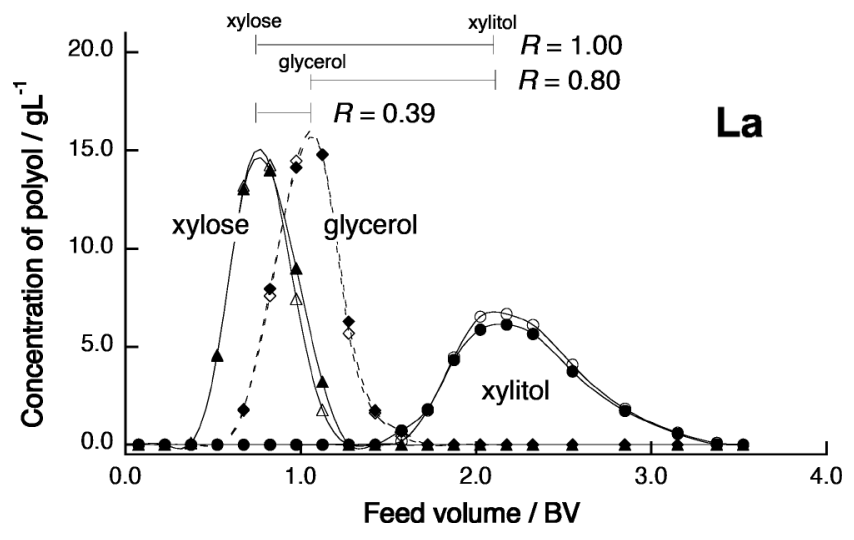

Fig. 2 Polyols separation by ligand-exchange chromatography using $\mathrm{La}^{3+}$-loaded resin. The feed volumes of the eluent were plotted as the relative value to the column bed volume, (eluent volume)/ (column bed volume). Column, $10 \mathrm{~mL}$ of $\mathrm{La}^{3+}$-form wet resin (AG 50W-4X; Bio-Rad); column length, $8.8 \mathrm{~cm}$; sample, $3 \mathrm{~mL}$ of $20 \mathrm{~g} / \mathrm{L}$ for xylose, xylitol, and glycerol; eluent, water; flow rate, $20 \mathrm{~mL} / \mathrm{h}$; temperature, $25^{\circ} \mathrm{C}$.
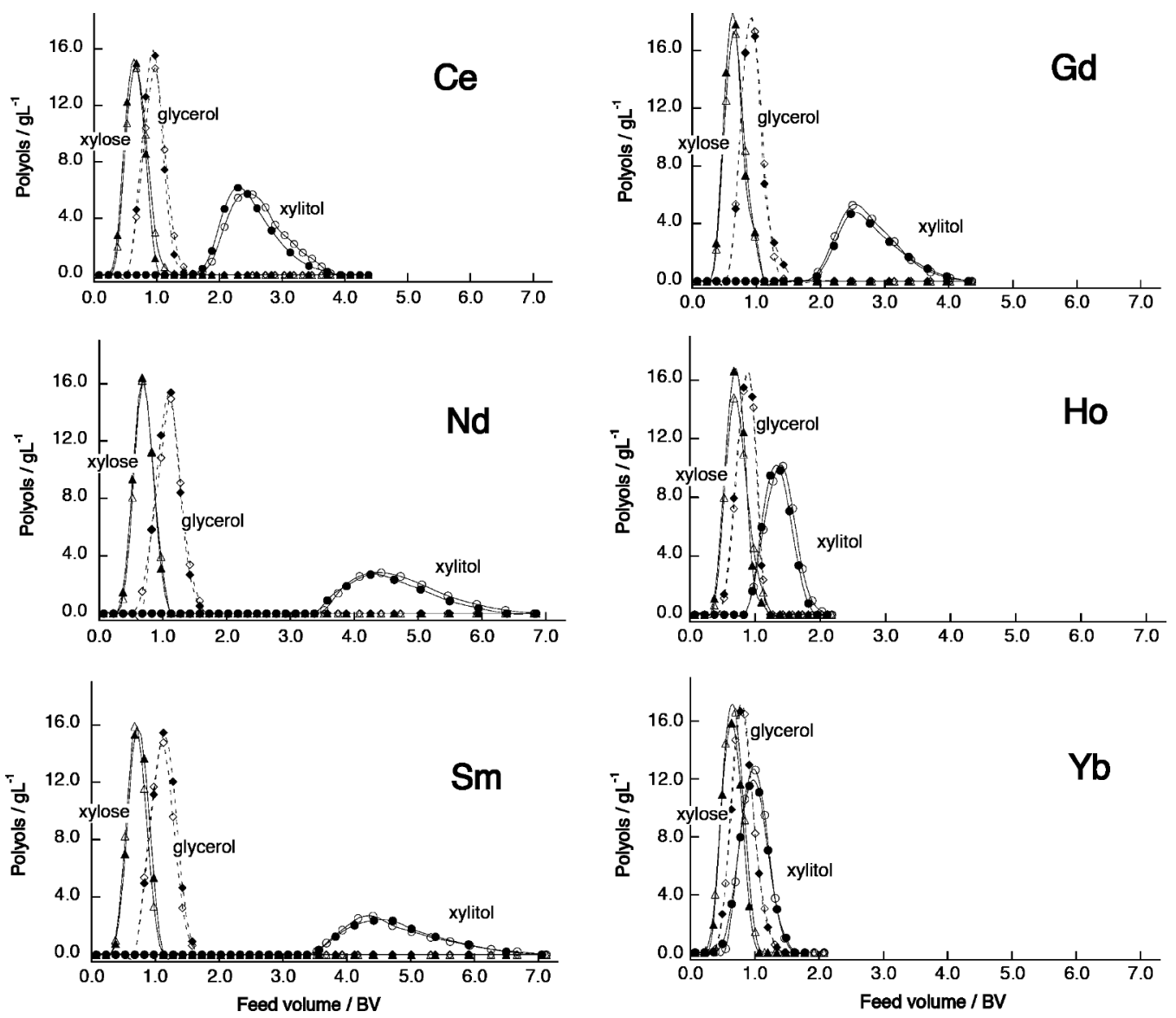

Polyols separation

The mixture of the main byproducts in bioethanol production processes from the bamboo tree, xylose, xylitol, and glycerol, was applied to chromatographic separation by the ligandexchange mode using a series of $\mathrm{Ln}^{3+}$-loaded adsorbents. The chromatograms for the polyol separation using $\mathrm{La}^{3+}$-loaded resins are shown in Fig. 2. The retention of xylitol was most strong, followed by glycerol and xylose in this order. Compared with glycerol having three hydroxyl groups, a five-hydroxyl group sequence on the linear flexible backbone of xylitol seems to effectively (at least transiently) coordinate to the immobilized $\mathrm{La}^{3+}{ }^{30}$ However, it is apparent that four hydroxyl groups in xylose seem not to coordinate to $\mathrm{La}^{3+}$ effectively, because xylose retention was weaker than that of glycerol. In the rigid pyranose structure of xylose, any three neighboring hydroxyl groups are not allowed to direct the same side of the structural backbone, making it difficult to form a stable complex with $\mathrm{La}^{3+} .^{31,32}$ Actually, the Morel group showed a significant difference in the thermodynamic parameters in the interaction of a pair of the epimers, ribose and arabinose, with $\mathrm{Ln}^{3+} .33,34$ The results observed here show that not only the number, but also the

Fig. 3 Polyols separation by ligand-exchange chromatography using $\mathrm{Ln}^{3+}$-loaded resins. All chromatographic conditions are the same as those shown in Fig. 2. 


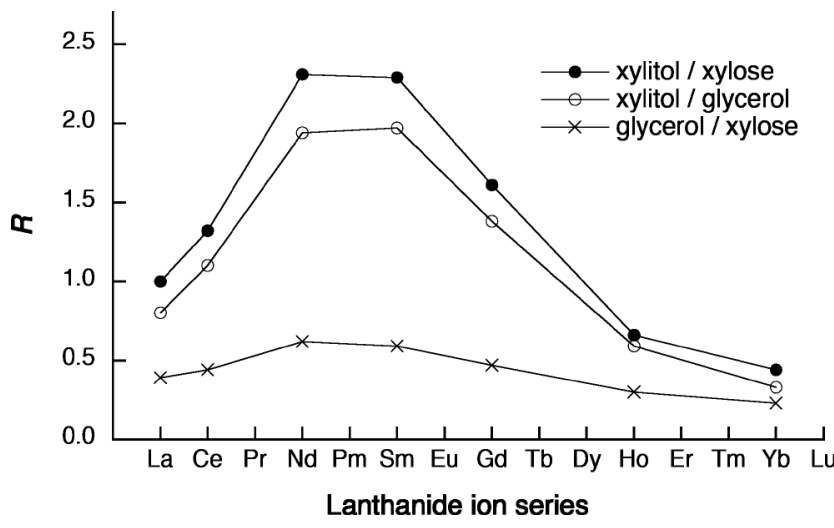

Fig. 4 Resolution $(R)$ of the two polyols by ligand-exchange chromatography using the adsorbents loaded a series of lanthanide ions (closed circle, xylitol/xylose; open circle, xylitol/glycerol; $x$, glycerol/xylose).

relative directions (stereochemistry) of hydroxy groups are critical for the formation of stable $\mathrm{La}^{3+} /$ sugar complexes.

The $R$ values between the two components were calculated to be $1.00,0.80$, and 0.39 for xylitol/xylose, xylitol/glycerol, and glycerol/xylose, respectively. That is, xylitol was nearly completely isolated from xylose and glycerol by almost the baseline separation using the short column. The results coincide with those reported by research groups of Gaset $^{22}$ and Stankovic. ${ }^{30}$ They reported on the chromatographic separation of a series of carbohydrates and alditols by a ligand-exchange mode using $\mathrm{La}^{3+}-$ loaded resins, respectively. Sugar alcohols, such as galactitol, xylitol, and arabinitol, with a linear backbone showed strong retention on the resin.

The adsorbents loaded with six other lanthanide ions were subjected to ligand-exchange chromatography for the polyol mixture in the same way. The obtained chromatograms are shown in Fig. 3. Reproducibility was observed for all of the adsorbents, showing negligible leakage of $\mathrm{Ln}^{3+}$. Interestingly, each of the $\mathrm{Ln}^{3+}$-loaded resins provided quite different elution profiles, especially for the retention volume $\left(V_{R}\right)$ of xylitol. The change in $V_{\mathrm{R}}$ or $R$ does not apparently follow the trend of lanthanide contraction. The $R$ values for the two components are plotted toward the atomic weight of the lanthanide series in Fig. 4. The results show the maximum at medium lanthanideloaded resins, such as of $\mathrm{Nd}^{3+}$ and $\mathrm{Sm}^{3+}$, and no significant retention was observed for the resins loaded with the heavier $\mathrm{Ln}^{3+}$. Aside from separation of the byproducts from bioethanol production, it would be worth for $\mathrm{La}^{3+}$-loaded resins to be subjected to systematic studies concerning polyol separation as general adsorbents with tunable retention.

It is known that the thermodynamic parameters of lanthanide complexation with various ligands show a nonlinear pattern as the function of the atomic numbers. Many factors should be considered to completely explain this unique behavior. One of the important ones would be desolvation. Complexation is the process of displacement of the hydrated water molecules on the metal ion by hydroxyl groups of analytes. Studies of X-ray diffraction, ${ }^{35}$ neutron scattering, ${ }^{36}$ and Raman spectra ${ }^{37}$ showed that the inner-sphere hydration number changes within the lanthanide ion series. The coordination number of the lanthanides is nine for the lighter ones $\left(\mathrm{La}^{3+}-\mathrm{Nd}^{3+}\right)$ and eight for the heavier ones $\left(\mathrm{Tb}^{3+}-\mathrm{Lu}^{3+}\right)$. This would be a main factor for explaining the irregularity in complexation at or around $\mathrm{Gd}^{3+}$, which is known to be a so-called "gadolinium break". ${ }^{38}$
The bell-shaped profile of the $R$ value shown in Fig. 4 looks similar to the stability constant profile already reported for the complexation of ribose with a series of lanthanide ions in aqueous solution. ${ }^{33}$ It shows that the gadolinium break is also true in ligand-exchange chromatography for the separation of polyols. That is, although a part of the coordination sphere of $\mathrm{Ln}^{3+}$ is tightly occupied by sulfonates on the resin, the unique character of the lanthanide series is still preserved in the residual room for coordination. This would be important knowledge for the design of adsorbents for the separation of carbohydrates.

\section{Conclusions}

As far as we know, this is the first report concerning the gadolinium break observed in systematic studies of ligandexchange chromatography for biomolecules separation. While the ion-exchange rates of each of $\mathrm{Ln}^{3+}$ slightly depend on its ionic radius (Table 1), the methods for adsorbent preparation are identical for all $\mathrm{Ln}^{3+}$. The leakage of metal ions during chromatography was negligible for all $\mathrm{Ln}^{3+}$ used in this study. Under these well-specified or-arranged conditions, we can tune the retention of the analytes by choosing the appropriate $\mathrm{Ln}^{3+}$ for given mixtures. Therefore, $\mathrm{Ln}^{3+}$-loaded resins would be an effective and flexible adsorbent for the separation of biomolecules, especially with the functional groups of hard Lewis bases. The results obtained in this study would be a useful guideline for designing the systems of ligand-exchange chromatography for biomolecules in water.

\section{Acknowledgements}

This work was partially supported by the Low Carbon Technology Research and Development Program, Ministry of the Environment, Japan (2010 - 2012).

\section{References}

1. A. Singh and G. P. Rangaiah, Ind. Eng. Chem. Res., 2017, 56, 5147.

2. H. B. Aditiya, T. M. I. Mahlia, W. T. Chong, Hadi Nur, and A. H. Sebayang, Renew. Sust. Energ. Rev., 2016, 66, 631.

3. L. Rocha-Meneses, M. Raud, K. Orupold, and T. Kikas, Agnon. Res., 2017, 15, 830.

4. K. Robak and M. Balcerek, Food Technol. Biotechnol., 2018, 56, 174.

5. Q.-F. Xu, C.-F. Liang, J.-H. Chen, Y.-C. Li, H. Qin, and J. J. Fuhrmann, Glob. Energ. Conserv., 2019, 21, e00787.

6. S. Suzuki, Plant Species Biol., 2015, 30, 63.

7. K. Okutomi, S. Shinoda, and H. Fukuda, J. Veg. Sci., 1996, 7, 723.

8. Z. Y. Sun, Y. Q. Tang, T. Iwanaga, T. Sho, and K. Kida, Bioresour. Technol., 2011, 102, 10929.

9. Z. Y. Sun, Y. Q. Tang, S. Morimura, and K. Kida, Bioresour. Technol., 2013, 128, 87.

10. M. Miura, I. Watanabe, Y. Shimotori, M. Aoyama, Y. Kojima, and Y. Kato, Wood Sci. Technol., 2013, 47, 515.

11. W. G. Morais Junior, T. F. Pacheco, D. Trichez, J. R. M. Almeida, and S. B. Goncalves, Yeast, 2019, 36, 349.

12. D. Camargo, L. Sene, D. I. L. S. Variz, and M. D. S. de Almeida Felipe, Appl. Biochem. Biotechnol, 2015, 175, 3628.

13. M. Miura, Y. Shimotori, H. Nakatani, A. Harada, and 
M. Aoyama, Appl. Biochem. Biotechnol, 2015, 176, 947.

14. L. Xu, L. Liu, S. Li, W. Zheng, Y. Cui, R. Liu, and W. Sun, Sugar Tech, 2019, 21, 341.

15. X. Zhu and A. Jyo, Sep. Sci. Technol., 2001, 36, 3175.

16. X. Zhu and A. Jyo, Water Res., 2005, 39, 2301.

17. Md. R. Awual, A. Jyo, M. Tamada, and A. Katakai, J. Ion Exchange, 2007, 18, 422.

18. Md. R. Awual, M. A. Shenashen, T. Yaita, H. Shiwaku, and A. Jyo, Water Res., 2012, 46, 5541.

19. Md. R. Awual, A. Jyo, T. Ihara, N. Seko, M. Tamada, and K. T. Lim, Water Res., 2011, 45, 4592.

20. Md. R. Awual, S. A. El-Safty, and A. Jyo, J. Environ. Sci., 2011, 23, 1947.

21. T. Ihara, T. Mitsuru, Y. Kitamura, Y. Chikaura, and A. Jyo, Anal. Sci., 2001, 17, i1229.

22. H. Caruel, L. Rigal, and A. Gaset, J. Chromatgr., 1991, $558,89$.

23. M. Stefansson and D. Westerlund, J. Chromatogr. A, 1996, $720,127$.

24. H.-T. Feng, X.-Y. Huang, C.-P. Luo, and M. M. Lee, J. Liq. Chrom. Rel. Technol., 2009, 32, 210.

25. R. Konradi and J. Rühe, Macromolecules, 2005, 38, 4345.

26. R. Kawamura, M. Satou, T. Yonesaka, and A. Yuchi, Anal. Sci., 2019, 35, 141.
27. S. Yoshii, M. Mori, D. Kozaki, T. Hosokawa, and $\mathrm{H}$ Itabashi, Anal. Sci., 2019, 35, 1117.

28. I. Suzuki, Y. Kumai, M. Kitagawa, Y. Kishimoto, K. Umegaki, T. Chiba, and J. Takebayashi, Anal. Sci., 2019, $35,1269$.

29. S. Kagaya, R. Ikeda, T. Kajiwara, M. Gemmei-Ide, and Y. Inoue, Anal. Sci., 2019, 35, 413.

30. L. Petrus, V. Bilik, L. Kuniak, and L. Stankovic, Chem. Zvesti., 1980, 34, 530 .

31. S. J. Angyal, Aust. J. Chem., 2000, 53, 567.

32. D. Goto, K. Ouchi, M. Shibukawa, and S. Saito, Anal. Sci., 2015, 31, 1143.

33. N. Morel-Desrosiers and J.-P. Morel, J. Chem. Soc., Faraday Trans., 1989, 85, 3461.

34. N. Morel-Desrosiers, C. Lhermet, and J.-P. Morel, J. Chem. Soc., Faraday Trans., 1993, 89, 1223.

35. A. Habenschuss and F. H. Spedding, J. Chem. Phys., 1980, 73, 442 .

36. C. Cossy, A. C. Barnes, J. E. Enderby, and A. E. Merbach, J. Chem. Phys., 1989, 90, 3254.

37. H. Kanno, J. Phys. Chem., 1988, 92, 4232.

38. G. Schwarzenbach and P. Gut, Helv. Chim. Acta, 1956, 39, 1589. 